Please do not remove this page

RMIT

UNIVERSITY

\title{
Operational Modal Analysis of a wing excited by transonic flow
}

Neu, Eugen; Jansen, Frank; Afaghi Khatibi, Akbar; Braun, Carsten; Orifici, Adrian

https://researchrepository.rmit.edu.au/esploro/outputs/9921862546501341/filesAndLinks?institution=61RMIT_INST\&index=null

Neu, E., Jansen, F., Afaghi Khatibi, A., Braun, C., \& Orifici, A. (2016). Operational Modal Analysis of a wing excited by transonic flow. Aerospace Science and Technology, 49, 73-79.

https://doi.org/10.1016/j.ast.2015.11.032

Document Version: Accepted Manuscript

Published Version: https://doi.org/10.1016/j.ast.2015.11.032

Repository homepage: https://researchrepository.rmit.edu.au

(c) 2015 Elsevier Masson SAS

Downloaded On 2023/04/26 17:43:49 +1000

Please do not remove this page 
Thank you for downloading this document from the RMIT Research Repository.

The RMIT Research Repository is an open access database showcasing the research outputs of RMIT University researchers.

RMIT Research Repository: http://researchbank.rmit.edu.au/

\section{Citation:}

Neu, E, Jansen, F, Afaghi Khatibi, A, Braun, C and Orifici, A 2016, 'Operational Modal Analysis of a wing excited by transonic flow', Aerospace Science and Technology, vol. 49, pp. 73-79.

See this record in the RMIT Research Repository at:

https://researchbank.rmit.edu.au/view/rmit:35749

Version: Accepted Manuscript

Copyright Statement: (c) 2015 Elsevier Masson SAS Creative Commons Attribution-NonCommercial-NoDerivatives 4.0 International License.

Link to Published Version:

http://dx.doi.org/10.1016/j.ast.2015.11.032 


\title{
Operational Modal Analysis of a Wing Excited by Transonic Flow
}

\author{
Eugen Neu ${ }^{\mathrm{a}}$, Frank Janser ${ }^{\mathrm{a}}$, Akbar A. Khatibi ${ }^{\mathrm{b}}$, Carsten Braun ${ }^{\mathrm{a}}$, Adrian C. Orifici ${ }^{\mathrm{b}}$ \\ ${ }^{a}$ FH Aachen UAS, Hohenstaufenallee 6, Aachen, Germany \\ ${ }^{b}$ RMIT University, GPO Box 2476 Melbourne, Victoria Australia, 3001
}

\begin{abstract}
Operational Modal Analysis (OMA) is a promising candidate for flutter testing and Structural Health Monitoring (SHM) of aircraft wings that are passively excited by wind loads. However, no studies have been published where OMA is tested in transonic flows, which is the dominant condition for large civil aircraft and is characterised by complex and unique aerodynamic phenomena. We use data from the HIRENASD large-scale wind tunnel experiment to automatically extract modal parameters from an ambiently excited wing operated in the transonic regime using two OMA methods: Stochastic Subspace Identification (SSI) and Frequency Domain Decomposition (FDD). The system response is evaluated based on accelerometer measurements. The excitation is investigated from surface pressure measurements. The forcing function is shown to be non-white, nonstationary and contaminated by narrow-banded transonic disturbances. All these properties violate fundamental OMA assumptions about the forcing function. Despite this, all physical modes in the investigated frequency range were successfully identified, and in addition transonic pressure waves were identified as physical modes as well. The SSI method showed superior identification capabilities for the investigated case. The investigation shows that complex transonic flows can interfere with OMA. This can make existing approaches for modal tracking unsuitable for their application to aircraft wings operated in the transonic flight regime. Approaches to separate the true physical modes from the transonic disturbances are discussed.
\end{abstract}

Keywords: operational modal analysis; modal parameters; flutter; structural health monitoring; HIRENASD; transonic

\section{Introduction}

In recent years significant progress has been made in developing and refining modal parameter identification methods that use environmental loads as the primary source

Email addresses: neu@fh-aachen.de (Eugen Neu), janser@fh-aachen.de (Frank Janser), akbar.khatibi@rmit.edu.au (Akbar A. Khatibi), c.braun@fh-aachen.de (Carsten Braun), adrian.orifici@rmit.edu.au (Adrian C. Orifici)

Preprint submitted to Aerospace Science and Technology 
of structural excitation. Those methods are today known under the name Operational 5 Modal Analysis (OMA) [1]. Modal parameters identified with OMA have been shown to be suitable for Structural Health Monitoring (SHM) of large civil engineering structures [2]. However, only limited publications have investigated the application of similar techniques to aircraft wings, and there is a lack of knowledge across a range of critical areas.

10 Abdelghani et al. [3] investigated an output-only subspace-based damage detection algorithm using a Paris MS760 airplane in a Ground Vibration Test (GVT). They showed that it is possible to detect small mass $(2 \%)$ and stiffness changes (blocked and released ailerons). The structure was artificially excited at two points with random white signals. Mevel et al. [4], Debille and Peeters [5] and Peeters et al. [6] extracted natural frequencies

15 and damping ratios from in-flight data, thereby showing that it is essentially possible to track some modal parameters during aircraft operation. However, they only published limited quantitative results and no informations about the flight conditions.

The application of OMA to transonic flow has not been previously demonstrated. This is significant as transonic flow not only involves complex aerodynamic phenomena, but

20 is the dominant flight regime for large passenger transport aircraft. As such, the performance of the various OMA techniques and their associated autonomous mode detection algorithms has not been characterised. For example, Stochastic Subspace Identification (SSI) is considered to be one of the most powerful parametric time-domain system identification methods [7], which was investigated in a variety of output-only damage

25 detection or SHM studies [2][8]. The vast majority of hitherto proposed SSI-based automatic modal parameter extraction methods for SSI try to automatize the interpretation of the consistency or stabilization diagram [9]. In contrast, Frequency Domain Decomposition (FDD) is a non-parametric frequency-domain method, which is an extension of the classic Peak Picking (PP) approach and was studied for damage detection and

30 SHM as well [8][10]. Modal parameters are automatically detected by searching for local maxima of the first singular value and subsequently checking the modal coherence in the vicinity of the peaks [11]. It is not known how the different mathematical foundations of automatic SSI and FDD perform for transonic flow excitation and whether there are any limitations of the techniques within this unique context. In addition, no wing-based

35 OMA publications have used input load measurements to gain critical insight into the aerodynamic excitation.

This study focuses on modal parameter extraction from a large-scale wind tunnel wing model excited by transonic flow. Modal parameters (natural frequencies, mode shapes and damping ratios) are extracted from dynamic measurements of the High Reynolds

40 Number Aerostructural Dynamics (HIRENASD) wind tunnel model using two OMAtechniques: FDD and SSI. Previous studies have shown that a periodic pressure wave builds at the surface of the HIRENASD wing, when operated in the transonic flow regime [12]. The properties of this transonic phenomenon and its interaction with the elastic structure are studied in detail in this work within the context of the OMA tech-

45 niques. The identified natural frequencies are compared to existing Experimental Modal Analysis (EMA) and Finite Element Method (FEM) results. The Angle Of Attack (AOA)-variability of the identified modal properties as well as the AOA-variability of the encountered transonic pressure waves are examined. These findings are assessed in 
the context of SHM for aircraft structures.

\section{Methods}

\subsection{HIRENASD model and data}

A dataset from the HIRENASD wind tunnel experiment is used for the present investigation. The HIRENASD project was a study of an elastic wing model in the transonic regime carried out in 2006. The tests were conducted at the European Transonic

${ }_{55}$ Wind tunnel (ETW). The aeroelastic behavior of a fixed-wing model was investigated at Reynolds and Mach numbers that are typically encountered by large aircraft in cruise flight. The wing was equipped with 9 functional accelerometers whose positions are shown in figure 1a and 205 functional pressure sensors distributed over 7 span-wise sections, which are shown in figure $1 \mathrm{~b}$.

${ }_{60}$ The $1.3 \mathrm{~m}$ semispan wing model was developed with Mach-number and Reynolds-number similarity in mind. The structure was designed to withstand the high aerodynamic loads and, at the same time, to allow for well measurable deformations. Other design goals were well separated fundamental eigenmodes, the ability to artificially excite the structure in a wide frequency range and operability under cryogenic conditions. According to Korsch et al. [13]

65 the desired static and dynamic system properties were attained in an iterative design process using academic and commercial finite element analysis and fluid-structure interaction tools. Detailed information about the experiment, the design goals and the iterative process of development were published in $[12,13]$.

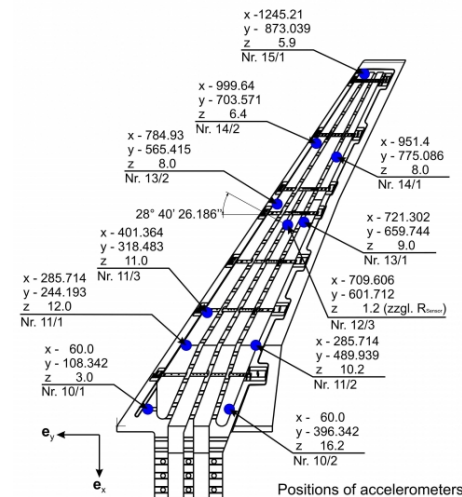

(a) Acceleration sensor positions.

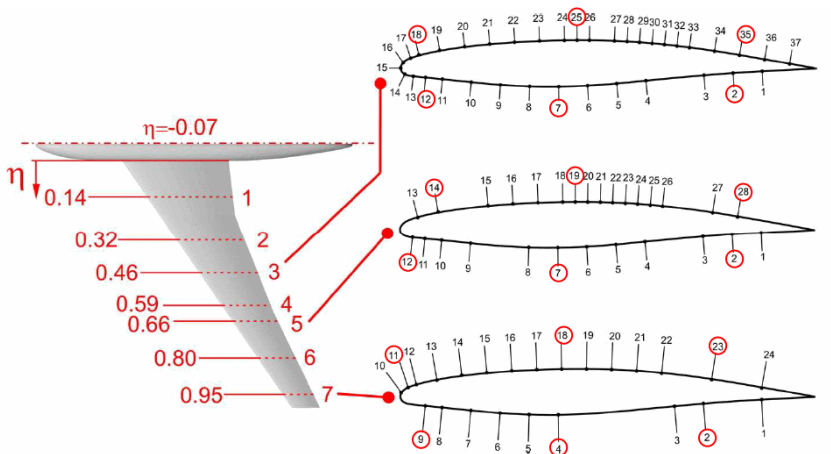

(b) Pressure sensor positions. Only the measurements from the circled pressure sensors are used in this investigation.

Figure 1: Accelerometer and pressure sensor positions and labels (adapted from [14]).

In this study results from a single 41 second measurement at a fixed operation point are investigated. The dataset was recorded at $279 \mathrm{~K}$ total temperature and $136 \mathrm{kPa}$ total pressure. The freestream Mach number was set to 0.8 resulting in a mean chord-based Reynolds number of $7 \times 10^{6}\left(c_{r e f}=0.3445 \mathrm{~m}\right)$. The AOA was slowly changed during 


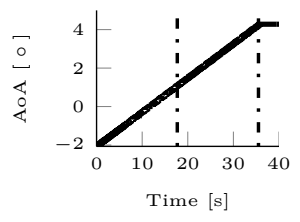

Figure 2: AOA time-history.

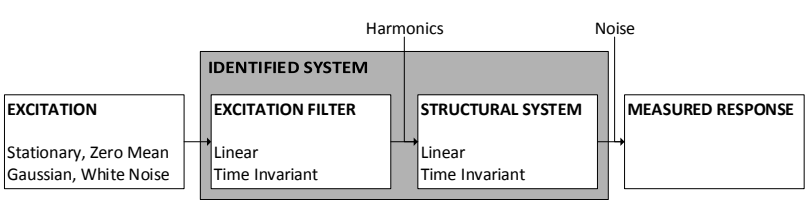

Figure 3: System identification from output-only measurements.

the recording window from $-2.1^{\circ}$ to $+4.2^{\circ}$ at a rate of change of $\approx 0.18^{\circ} / \mathrm{s}$ (figure 2 ). The dataset was separated into two non-overlapping blocks with a duration of 17.95

75 seconds each. The split was performed to confine the non-stationary influence of the AOA-sweep and to be able to assess the variation of the identification process. The block size is chosen to include approximately 500 recurrences of the lowest natural frequency to allow for a statistically valid identification. Additional investigations with a further subdivided dataset were conducted to examine the natural frequency evolution with increasing AOA.

Modal parameters were extracted using the commercial software ARTeMIS Modal [15]. Linear trends were removed, the data were processed through a low-pass filter with a cut-off frequency of $480 \mathrm{~Hz}$ and subsequently decimated to a sampling rate of $1000 \mathrm{~Hz}$. The resolution for the Curve-fit Frequency Domain Decomposition (CFDD) spectral

85 density estimation was set to 2048 lines in the investigated frequency range. Only modes that were automatically detected by the investigated algorithms are presented throughout the paper, thus the suitability of the investigated techniques for automatic OMA can be assessed.

The pressure measurements were contaminated by alternating current disturbances at 90 multiples of $100 \mathrm{~Hz}(200 \mathrm{~Hz}, 300 \mathrm{~Hz}, \ldots)$. The affected frequency lines were replaced by least square fits through the neighboring frequency lines in all affected figures.

\subsection{Operational Modal Analysis}

In general the stochastic unobserved load in OMA is assumed to be stationary, ergodic and zero mean during the derivation of the investigated OMA methods. Furthermore the

95 input load is assumed to have a constant Power Spectral Density (PSD) in the frequency range of interest (band-limited white noise) [16]. Figure 3 shows the relationship between the presumed excitation and the measured response. Any deviation from the required white excitation can be represented through an excitation filter, if the excitation can be represented by a linear, time-invariant system. System identification with OMA will

100 then identify a joined system consisting of the excitation filter response and the structural system response. The interaction between the excitation and the structural system is a key focus of this work and is discussed in detail in section 3.1 and 3.2.

\subsubsection{Stochastic Subspace Identification}

Several studies showed that SSI can be considered to be one of the most sophisticated

105 OMA-techniques available today [16][17]. The idea of the approach is to extract a statespace representation of the linear, time-invariant physical model by orthogonal projection 
of block Hankel matrices, followed by Singular Value Decomposition (SVD) and regression.

The major steps in SSI are to arrange the individual discrete signals $y_{l}[n]$ into a single data matrix $Y[n]=\left[y_{1}[n], y_{2}[n], \cdots, y_{L}[n]\right]^{T}$. Then the data matrix $Y$ is rearranged into a Block Hankel matrix $H$.

$$
H=\left[\begin{array}{l}
Y[:, 1: N-2 s] \\
Y[:, 2: N-2 s+1] \\
\cdots \\
Y[:, 2 s: N]
\end{array}\right]=\left[\begin{array}{l}
H_{p} \\
H_{f}
\end{array}\right]
$$

The orthogonal projection of the future $H_{f}$ into the past $H_{p}$ produces the projection matrix $O$, where $2 s$ is the total data shift.

$$
O=H_{f} H_{p}^{T}\left(H_{p} H_{p}^{T}\right)^{-1} H_{p}
$$

$O$ is further decomposed using SVD.

$$
O=\left[\begin{array}{ll}
U_{1} & U_{2}
\end{array}\right]\left[\begin{array}{cc}
S_{1} & 0 \\
0 & 0
\end{array}\right]\left[\begin{array}{c}
V_{1}^{T} \\
V_{2}^{T}
\end{array}\right]
$$

$U_{1} S_{1}^{\frac{1}{2}}$ and $S_{1}^{\frac{1}{2}} V_{1}^{T}$ can be related to the state space representation of a linear, timeinvariant discrete time physical system

$$
\begin{aligned}
x[n+1] & =A x[n]+w[n] \\
y[n] & =C x[n]+v[n]
\end{aligned}
$$

115 where $x[n]$ is the discrete state space vector, $A$ is the dynamic stiffness matrix, which completely describes the dynamic system. $C$ is the output matrix, which describes the transformation of the internal state $x[n]$ to the measurements $y[n] . w[n]$ and $v[n]$ are unmeasured stationary, zero mean, white noise vectors.

The output matrix estimate $\hat{C}$ can immediately be extracted from the observability 120 matrix by taking the first $L$ rows from $U_{1} S_{1}^{\frac{1}{2}}$ and the system matrix estimate $\hat{A}$ can be extracted from the observability matrix by blockwise regression.

Finally, estimates for natural frequencies $f_{i}$, damping ratios $\xi_{i}$ and mode shapes $\phi_{i}$ can be extracted from $\hat{A}$ using an eigenvalue decomposition

$$
\hat{A}=\Psi\left[\mu_{i}\right] \Psi^{-1}
$$

and proper scaling $\Phi=\hat{C} \Psi, \lambda_{i}=1 / T \cdot \ln \left(\mu_{i}\right), \omega_{i}=\left|\lambda_{i}\right|$ and $\xi_{i}=\operatorname{Re}\left(\lambda_{i}\right) /\left|\lambda_{i}\right|$. 


\subsubsection{Frequency Domain Decomposition}

The second OMA method used to extract modal parameter from the HIRENASD exper-

imental data is Curve-fit Frequency Domain Decomposition. FDD was first presented by Brincker et al. [18]. The basic idea is to decompose the Cross Spectral Density Matrix (CSDM) estimate $\hat{G}_{y}$ using SVD.

$$
\hat{G}_{y}[\omega]=U[\omega] S[\omega] V^{*}[\omega]
$$

For lightly damped structures only a limited number of modes (typically one or two) will significantly contribute to the system response at a certain frequency $\omega_{k}$. Therefore, in 130 the vicinity of the $k$ th natural angular frequency $\omega_{k}$, the first singular vector $u_{1}\left[\omega_{k}\right]$ is an estimate of the corresponding mode shape $\hat{\phi}_{k}$. The first singular value $s_{1}[\omega]$ can then be interpreted as PSD of the Single Degree-of-Freedom (SDOF) system [18].

With the classic FDD approach it is only possible to manually estimate natural frequencies and mode shapes. A technique to estimate damping ratios in the time-domain was later proposed by Brincker et al. [19] and today is known as Enhanced Frequency Domain Decomposition (EFDD). Jacobsen et al. [20] proposed the CFDD algorithm, which relocates the damping estimation process back to the frequency domain, thereby improving the natural frequency and damping ratio estimation.

\section{Results and Discussion}

\subsection{The turbulent and transonic wind excitation}

The large number of pressure gauges at the surface of the HIRENASD wing allows for a detailed investigation of the input loads. Traditionally the input loads are not measured in OMA but rather are represented by the model described in section 2.2. Wind excitation is assumed to perfectly obey the OMA assumptions [21]. In this section it is discussed in 145 what respect the real transonic wind loads deviate from the ideal stochastic model and how these deviations influence the modal parameter identification.

Figure 4 shows $c_{\mathrm{p}}$-PSD-estimates from four surface pressure sensors (see figure 4 for labels and figure $1 \mathrm{~b}$ for locations). The wing was slowly rotated during the measurement period. As a consequence the surface pressure distribution and power vary with time and the OMA ergodicity and stationarity assumptions are not fulfilled. Benveniste and Mevel [22] showed that subspace algorithms will converge to the true eigenstructure despite nonstationaries in the excitation. Of course, the "true eigenstructure" may be velocity-dependent or may depend on the static preload of the structure, as in fact is the case for a wing operated at different velocities [23] or AOAs [24].

155 Strong and narrow-banded peaks at $129 \mathrm{~Hz}$ are visible in every PSD estimate. Additional peaks at $258 \mathrm{~Hz}$ and $387 \mathrm{~Hz}$ are visible in some PSD estimates as well. Ballmann et al. [12] showed the peak at $129 \mathrm{~Hz}$ to be a transonic phenomenon, which they call an upstream running pressure wave. The occurrence of additional or secondary pressure waves was not 

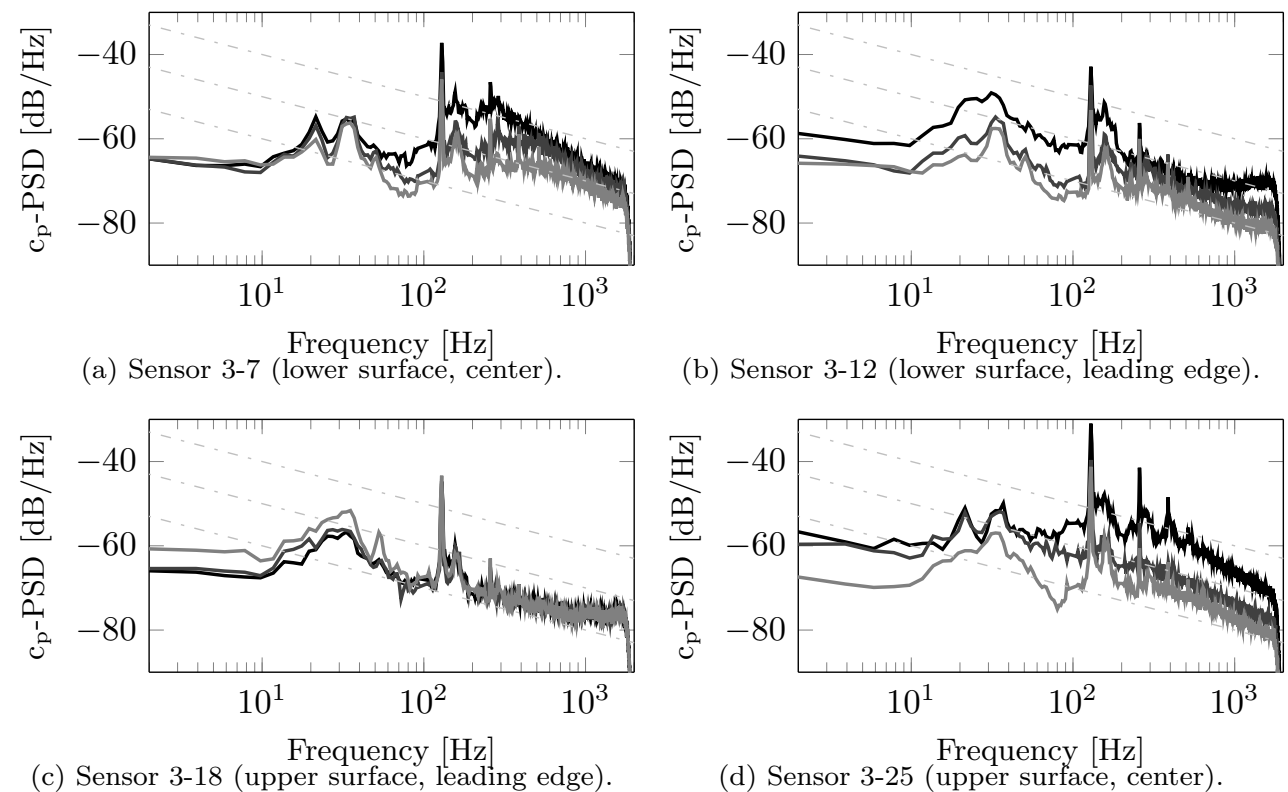

$$
\mathrm{AoA}-2^{\circ} \longrightarrow \mathrm{AoA}+1^{\circ} \longrightarrow \mathrm{AoA}+4^{\circ} \cdots \omega^{-1} \text {-trend }
$$

Figure 4: $\mathrm{c}_{\mathrm{p}}$-PSD content throughout the AOA-sweep . Dashed lines indicate an $\omega^{-1}$-trend.

reported. The SVD of the CSDM from 18 pressure sensors (the application of the FDD algorithm to the pressure data) reveals more of the true nature of the pressure wave (figure 5). The frequency investigated by Ballmann et al. [12] is in fact only the fundamental frequency of a family of pressure waves which occur at multiples of the fundamental pressure wave frequency. The SVD also reveals a second group of significantly weaker pressure waves, which have the same distance between each other $(129 \mathrm{~Hz})$ but are shifted against the previously described group by $27 \mathrm{~Hz}(156 \mathrm{~Hz}, 285 \mathrm{~Hz}, 414 \mathrm{~Hz}) .27 \mathrm{~Hz}$ also happens to be the first fundamental bending mode frequency of the wing and a strong and broad elongation can be seen in the SVD-diagram at $27 \mathrm{~Hz}$ as well. This indicates that a complex interaction between the eigenmovement of the system and the transonic flow seems to be at work and further investigation is necessary to reveal the true nature of this phenomenon.

The key conclusion from the analysis is that the occurrence of narrow-banded disturbances clearly violates the OMA band-limited white noise assumption. Hence, the excitation will be part of the identified system in accordance with figure 3 and the pressure waves will be identified as system poles. Whether the full system behavior of the pressure waves can be modelled using a linear, time-invariant filter is questionable. This question will be further evaluated in section 3.2, where the results of the modal identification are presented. 


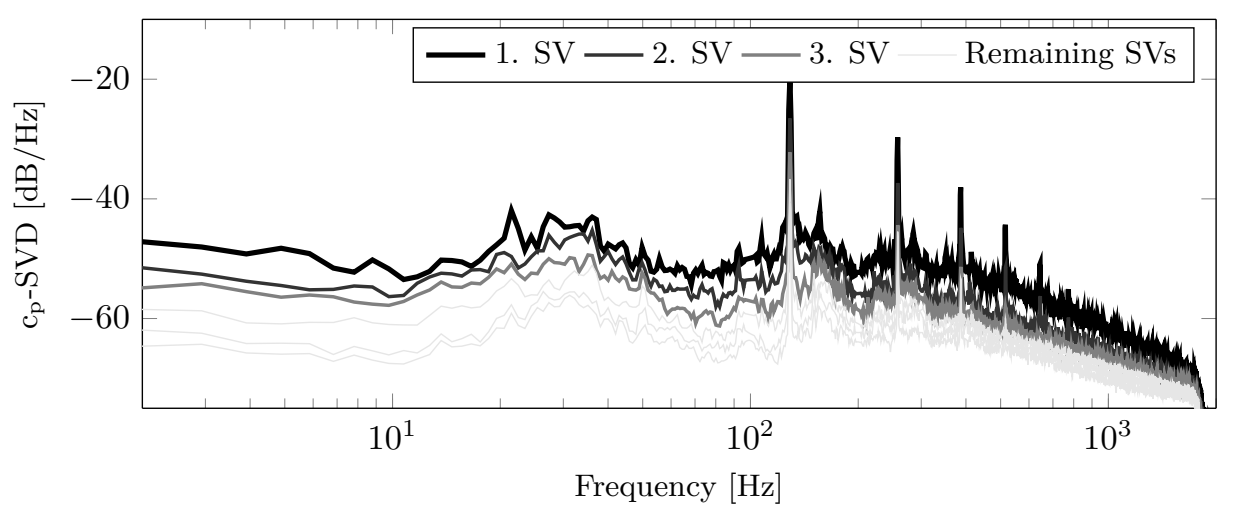

Figure 5: SVD of the CSDM from the 18 pressure sensors highlighted in figure $1 \mathrm{~b}$.

\subsection{Modal parameter identification - evaluation and analysis}

In table 1 OMA results are compared to hammer impact modal analysis data and the results of a numerical modal analysis. The wing was extensively tested using hammer impact, sine sweep and harmonic excitation EMA before deployment to ETW. The results have been published in Korsch et al. [13]. Natural frequencies and damping ratios obtained from the hammer impact tests are included in table 1. Furthermore, HIRENASD data are used as a test case in the Aeroelastic Prediction Workshop (AePW) [25]. In this context an already available FEM model was further enhanced and validated by Wieseman et al. [26]. The natural frequencies obtained from the numerical model are also included in table 1. The EMA and FEM results are associated with the modes extracted from OMA by natural frequency correlation and qualitative mode-shape comparison.

Table 1: Natural frequencies and damping ratios identified from accelerometer data using SSI, CFDD hammer impact test data from Korsch et al. [13] and FEM data from Wieseman et al. [26]. B stands for bending, $\mathrm{T}$ for torsion, $\mathrm{F}$ for a for-and-aft in-plane bending mode and $\mathrm{P}$ for a pressure-wave induced oscillation. MCF is the Mode Complexity Factor.

\begin{tabular}{rrrrrrrrrrrrr}
\hline & SSI acc 1-half & \multicolumn{4}{c}{ SSI acc 2 -half } & \multicolumn{4}{c}{ CFDD acc 2-half } & \multicolumn{2}{c}{ Impact } & FEM \\
\hline Mode & $f$ & $\xi$ & MCF & $f$ & $\xi$ & MCF & $f$ & $\xi$ & MCF & $f$ & $\xi$ & $f$ \\
& {$[\mathrm{~Hz}]$} & {$[\%]$} & {$[\%]$} & {$[\mathrm{Hz}]$} & {$[\%]$} & {$[\%]$} & {$[\mathrm{Hz}]$} & {$[\%]$} & {$[\%]$} & {$[\mathrm{Hz}]$} & {$[\%]$} & {$[\mathrm{Hz}]$} \\
1B & 26.61 & 3.16 & 0.01 & 27.13 & 3.01 & 0.00 & 27.24 & 3.21 & 0.00 & 25.75 & 0.07 & 25.55 \\
2B & 78.60 & 1.58 & 0.26 & 79.55 & 1.85 & 0.16 & 79.33 & 1.40 & 0.08 & 71.11 & 0.27 & 80.25 \\
1F & & & & 118.90 & 3.97 & 6.06 & & & & 111.66 & 0.34 & 106.19 \\
1P & & & & 128.74 & 0.46 & 84.85 & & & & & & \\
1P & 129.27 & 0.15 & 72.63 & 128.76 & 0.18 & 73.75 & & & & & & \\
2P & 160.76 & 1.88 & 14.99 & 156.45 & 3.94 & 28.42 & & & & & \\
3B & 168.81 & 1.98 & 0.37 & 167.33 & 2.24 & 0.96 & 167.67 & 0.94 & 0.44 & 149.34 & 0.71 & 160.35 \\
4B & 235.58 & 2.79 & 1.10 & 236.23 & 1.86 & 0.96 & 237.11 & 0.91 & 0.33 & & & 242.00 \\
2F & & & & 250.88 & 3.17 & 56.73 & & & & & \\
3P & 259.33 & 0.75 & 0.59 & 257.39 & 0.34 & 9.17 & 258.74 & 0.23 & 1.47 & & \\
1T & 266.22 & 1.03 & 0.86 & 264.98 & 1.40 & 0.13 & & & & & \\
5B & 349.26 & 2.88 & 11.00 & 345.70 & 1.57 & 3.22 & 364.45 & 0.06 & 11.49 & & & 354.15 \\
2T & 423.49 & 0.70 & 1.11 & 422.76 & 0.81 & 1.49 & 422.33 & 0.76 & 1.46 & & 0.26 & 271.88 \\
\hline
\end{tabular}

${ }_{190}$ The first row in table 1 points out the dominant type of movement for the respective mode shape. The data in the columns SSI acc 1-half, SSI acc 2-half and CFDD acc 2-half were identified from the first and the second half of the accelerometer dataset using the 
SSI and CFDD method. The FEM results correspond quite well with the observed OMA results. The only modes identified by OMA that could not be associated to FEM-modes are $1 \mathrm{P}, 2 \mathrm{P}$ and $3 \mathrm{P}$ but these can clearly be related to sharp peaks in the excitation spectra (figure 5). Hence, as anticipated in the discussion of section 3.1, the pressure waves are detected as part of the identified system. All natural frequencies identified under operational conditions in ETW are higher than the frequencies measured during the dynamic qualification tests described in Korsch et al. [13]. This indicates a stiffer clamping in ETW than under laboratory conditions. The damping obtained under operational conditions is dominated by aerodynamic forces and therefore is significantly higher than the damping measured during the hammer impact tests. The for-and-aft bending modes $(1 \mathrm{~F}, 2 \mathrm{~F})$ should not be detectable with the out-of-plane accelerometers but cross-axis sensor interference seems to be strong enough to induce a detectable output.

To investigate the AOA-variability the dataset was subdivided into four equally sized subsets and modal parameters were estimated for each individual set. Figure 6 shows the change in relative frequency for these four datasets. The individual natural frequencies are normalized to the frequency identified from the first subset. The errorbars indicate how strong the frequencies vary at different model orders in the stabilization diagram. Especially the lower natural frequencies show a significant AOA-dependency. The AOA-variability cannot be explained by linear aerodynamic or structural theory and most likely is the result of structural geometric nonlinearity. A modal analysis is always a linearization about the current system deformation state. The deformation introduced by the AOA-change seems to be large enough to cause a detectable change in natural

215 frequencies. This effect has been described for high-aspect-ratio wings, where it is significantly more pronounced [24] but will of course occur in any cantilever-like structure subjected to significant static deformation. A distinct AOA-dependency could not be detected for damping ratios, mode shapes or mode shape complexities.
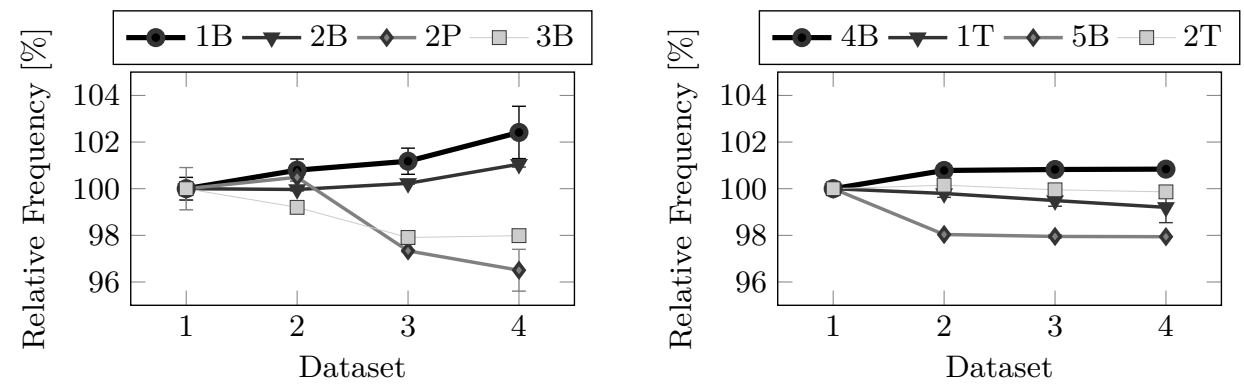

Figure 6: Evolution of natural frequency estimates with increasing AOA.

Only $2 \mathrm{P}$ shows a non-monotonic behavior in figure $6.1 \mathrm{P}$ and $3 \mathrm{P}$ were not added to the diagram because both modes split into multiple modes with increasing AOA, whereby the gap between the identified frequencies reaches $2 \mathrm{~Hz}$. The Mode Complexity Factors (MCFs) for all modes that are associated with pressure waves are more than an order of magnitude larger than the MCFs of the wing modes (table 1 ). The MCF will be $0 \%$ for real mode shapes and $100 \%$ for fully imaginary modes [15]. The detected pressure wave mode shapes change with increasing AOA, in contrast to the mode shapes of the wing (figure 7). According to Ballmann et al. [12] the area covered by the pressure wave 
and its amplitude significantly increases with AOA, which is in line with our findings of strongly changing mode shapes. These findings show that the pressure wave excitation has multiple non-linear characteristics and therefore cannot be represented by a linear, time-invariant system.

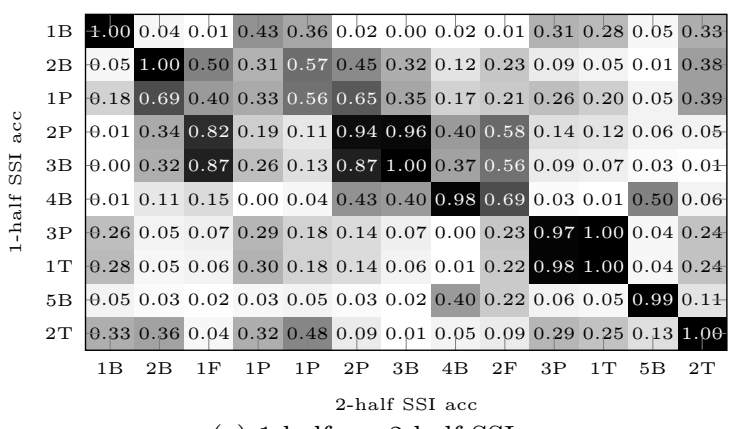

(a) 1-half vs. 2-half SSI

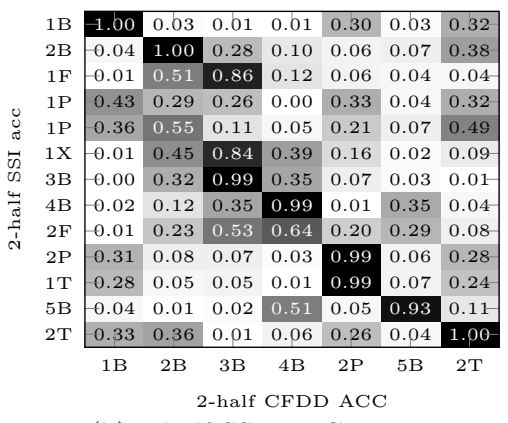

(b) 2-half SSI vs. CFDD

Figure 7: The visualization shows the Modal Assurance Criterion (MAC). A value (color) of 1 (black) means that the two mode shapes match exactly whereas a value of 0 (white) means that the mode shapes are completely uncorrelated.

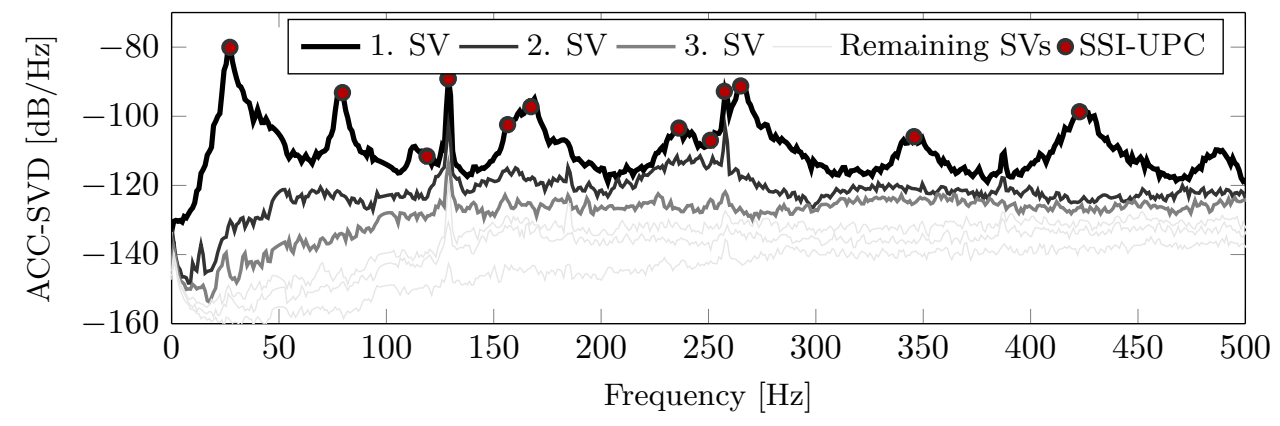

Figure 8: The figure shows Singular Value frequency-plots for accelerometer data.

\subsection{Automatic CFDD vs. SSI}

Table 1 shows a comparison between modes identified with SSI and CFDD. Significantly less modes have been automatically detected using CFDD. Figure $7 \mathrm{~b}$ shows that whenever mode shapes are detected by the CFDD method they are practically identical to the mode shapes identified with SSI. The only exception is the fifth bending mode $(5 \mathrm{~B})$ where the MAC is only 0.93 .

The automatic CFDD algorithm was not able to detect any of the pressure wave modes. CFDD relies on an automatic mode detection algorithm where singular vectors at neighbouring spectral lines are compared with the singular vector of the local first singular value maximum using the MAC [11]. $1 \mathrm{P}$ for example is not detected because the peak is very sharp and the singular vectors are changing rapidly throughout the frequency range. 
Figure 7a shows that the mode shapes of $3 \mathrm{P}$ and $1 \mathrm{~T}$ strongly interact and correlate and further investigation showed that the algorithm always selects the higher peak to be the single peak of the whole 3P-1T-region. The CFDD identification was also applied to the further subdivided accelerometer datasets and overall showed a less reliable identification behavior. $3 \mathrm{P}$ and $1 \mathrm{~T}$ were identified twice each but never both from the same dataset. $4 \mathrm{~B}$ was identified once, and 5B thrice. The investigated automatic SSI algorithm is based on the comparison of modes detected from different system orders [15]. This approach is actually not limited to SSI-methods but can be used with any parametric system identification method where the system order is used as a parameter [9]. This approach does not rely on a comparison at neighboring spectral lines and therefore allows the detection of very narrow-banded disturbances, as long as they are identified at multiple (or consecutive) system orders.

The findings here are consistent with the findings of another study where CFDD and the SSI method are compared for the identification of a wind-excited cantilever beam [27]. There it was found that the Stochastic Subspace Identification - Canonical Variate Analysis (SSI-CVA) method has superior mode detection capabilities for modes which are in the near proximity to other modes with significantly larger energy levels. The same was found to be true for weakly excited modes, that barely were above the noise floor.

260 The results here demonstrate that, in addition, automatic SSI-algorithms seem to be inherently better suited for the detection of very narrow-banded disturbances.

\subsection{Discussion}

In section 3.1 it was seen that the wind excitation is contaminated by pressure waves and in section 3.2 it was shown that some of these pressure waves are identified as part of the physical system. From the perspective of SHM this kind of effect needs to be separated from nearby real physical modes which are part of the damage-sensitive feature vector. The obvious solution is to measure the surface pressure at representative locations and separate physical and non-physical modes based on a partial input load measurement. Another way is to ignore modes with a significant MCF when extracting and comparing

270 damage-sensitive features. As aerodynamic damping is non-proportional and damage often results in a non-linear system behavior this possibility may limit the applicability of modal parameter-based damage assessment. A third solution is to mark modes with a damping ratio below a certain threshold as non-physical. Damping estimates for $1 \mathrm{P}$ and $3 \mathrm{P}$ are nearly an order of magnitude lower than the detected damping for the physical

275 modes. But this was not found to be true for $2 \mathrm{P}$ and there are flight conditions where the damping ratio of natural modes can become very small (i.e. flutter). Furthermore structural damage is known to alter the dissipate behavior of structures. Finally the singular value behavior for $1 \mathrm{P}$ and $3 \mathrm{P}$ in the SVD-plot shown in figure 8 significantly differs from the behavior of the surrounding modes. In contrast to the physical modes, 280 where only one or two singular values peak in the vicinity of the detected mode, for $1 \mathrm{P}$ and $3 \mathrm{P}$ all singular values show a local maxima. But again, this was not found to be true for $2 \mathrm{P}$.

In section 3.2 the natural frequency AOA-dependency was investigated. Studies where natural frequencies have been used for damage detection have shown that especially 
285 lower-natural frequencies have a very low sensitivity even to significant damage [28]. With respect to SHM for fixed-wings this means that either the AOA needs to be part of the operational and environmental variability considerations or that lower-natural modes should be skipped from the set of damage-sensitive features altogether. On the other hand, the nearly ideal MAC values between the 1-half and 2-half datasets in figure

2907 show that the MAC seems to be less sensitive to AOA-changes. This makes MAC and maybe other mode shape related indicators good damage-sensitive feature candidates for fixed-wing SHM.

There are important differences between the investigated model and an aircraft wing. First, the HIRENASD experiment was developed with Mach-number and Reynolds-

295 number similarity in mind. The structure was designed to withstand the high aerodynamic loads and to have clearly separated modes [13]. Thus, whereas real large aircraft have a high modal density in the very low frequency range (up to 16 elastic modes in $2 \mathrm{~Hz}$ according to Lau et al. [29]), the modes of the HIRENASD wing are well spread over a broad frequency band and are easier to detect. Second, there are fundamental differences

300 between the dynamic excitation in a wind tunnel and in-flight. The wind tunnel model is excited by boundary layer turbulence and facility-dependent freestream turbulence. An aircraft in-flight is excited by boundary layer turbulence and atmospheric turbulence. Information about the freestream turbulence in ETW was not available. Hence, it was not possible to separate the freestream contribution from the boundary layer contribution. No wing surface pressure measurements from a large aircraft in-flight are available in the open literature to address these aspects.

Despite the described differences, multiple key similarities exist between the investigated wind tunnel model and a large aircraft wing. The outer shape of the structure is identical, except for a scaling factor. In both cases the system in question is a cantilever-type structure. The excitation is comparable, if not identical. Based on these similarities and the findings in this study there is reasonable ground to presume that a high number of modal parameters can be extracted from output-only acceleration measurements of a large aircraft wing and that these parameters can be used as damage-sensitive features in $[. .1$ ]a SHM system.

\section{Conclusions}

Modal parameters were successfully and automatically extracted from the HIRENASD fixed-wing wind tunnel model, which was operated in the transonic flow regime.

Surface pressure measurements revealed the wind-excitation to be non-white, non-stationary and moreover to include strong and narrow-banded transonic pressure waves. These 320 disturbances dynamically interfered with the wing structure and were sometimes identified as structural system modes by the investigated OMA methods. Further investigation showed these pressure waves to be partially recognizable from output-only measurements by their very small damping ratio, the high mode shape complexity and the deviating singular value behavior when compared to real physical modes.

\footnotetext{
${ }^{1}$ removed: an
} 
325 Two different methods have been investigated for OMA: SSI and CFDD. A comparison with EMA and FEM data showed that only the SSI-method was able to detect all out-of-plane bending and torsional modes in the investigated frequency range. Furthermore, only the automatic SSI method was able to reliably detect the narrow-banded transonic pressure waves. The discussion showed this to be an inherent advantage of

330 automatic modal parameter identification methods that are based on parametric system identification methods, like SSI.

The wing model was slowly rotated throughout the measurement period. Natural frequencies were shown to have a dependency on AOA, particularly at lower frequencies, which was attributed to a structural geometric nonlinearity. Mode shapes, damping ra-

335 tios and mode shape complexities showed no distinct AOA-dependency. The pressure waves, on the other hand, were strongly influenced by the AOA and the system behavior was shown to be nonlinear in several ways.

\section{Acknowledgement}

We are very grateful to Prof. Dr. Ballmann and Dr. Boucke from RWTH Aachen Uni340 versity for providing us with the measurement data and for their help with interpreting those. The HIRENASD project was led by RWTH Aachen University (RWTH), support came from Airbus and the German Aerospace Center (DLR). The project was funded by the German Research Foundation (DFG).

\section{References}

[1] R. Brincker, P. H. Kirkegaard, Special issue on Operational Modal Analysis, Mechanical Systems and Signal Processing 24 (5) (2010) 1209-1212.

[2] F. Magalhães, A. Cunha, E. Caetano, Vibration based structural health monitoring of an arch bridge: From automated OMA to damage detection, Mechanical Systems and Signal Processing 28 (2012) 212-228.

350 [3] M. Abdelghani, M. Goursat, T. Biochini, On-Line Modal Monitoring of Aircraft Structures Under Unknown Excitation, Mechanical Systems and Signal Processing 13 (6) (1999) 839-853.

[4] L. Mevel, A. Sam, M. Goursat, Automated On-line Monitoring During a Flight, in: Proceedings of the 22nd IMAC Conference and Exposition on Structural Dynamics, 1147-1155, 2004.

[5] J. Debille, B. Peeters, The Benefits of Operational Modal Analysis of Aircraft and Spacecraft Structures, in: Proceedings of European Test \& Telemetry Conference, 1-6, 2005.

[6] B. Peeters, T. d. Troyer, P. Guillaume, In-flight modal analysis - a comparison between sweep and turbulence excitation, in: Proceedings of the 22th International Conference on Noise and Vibration Engineering, 1627-1641, 2006.

[7] R. Brincker, P. Andersen, Understanding Stochastic Subspace Identification, in: Proceedings of the 24th International Modal Analysis Conference, 461-466, 2006.

[8] B. Moaveni, A. R. Barbosa, J. P. Conte, F. M. Hemez, Uncertainty analysis of system identification results obtained for a seven-story building slice tested on the UCSD-NEES shake table, Structural Control and Health Monitoring .

[9] E. Reynders, J. Houbrechts, G. d. Roeck, Fully automated (operational) modal analysis, Mechanical Systems and Signal Processing 29 (2012) 228-250.

[10] C. Rainieri, G. Fabbrocino, E. Cosenza, Near real-time tracking of dynamic properties for standalone structural health monitoring systems, Mechanical Systems and Signal Processing 25 (8) (2011) 3010-3026. 
[11] R. Brincker, P. Andersen, N.-J. Jacobsen, Automated Frequency Domain Decomposition for Operational Modal Analysis, in: Proceedings of the 25th IMAC Conference and Exposition on Structural Dynamics, Society for Experimental Mechanics, 2544-2550, 2007.

[12] J. Ballmann, A. Boucke, B. Chen, L. Reimer, M. Behr, A. Dafnis, C. Buxel, S. Buesing, H. Reimerdes, M. Kordt, J. Brink-Spalink, F. Theurich, A. Buscher, Aero-Structural Wind Tunnel Experiments with Elastic Wing Models at High Reynolds Numbers (HIRENASD - ASDMAD), in: Proceedings of the 49th AIAA Aerospace Sciences Meeting, 1-17, 2011.

[13] H. Korsch, A. Dafnis, H.-G. Reimerdes, Dynamic qualification of the HIRENASD elastic wing model, Aerospace Science and Technology 13 (2-3) (2009) 130-138.

[14] J. Ballmann, A. Dafnis, HIRENASD website, URL http://heinrich.lufmech.rwth-aachen.de/, 2010 .

5] Structural Vibration Solutions A/S, ARTeMIS Modal: Documentation, URL http://www.svibs.com/products/ARTeMIS_Modal.aspx, 2013.

[16] E. Reynders, System Identification Methods for (Operational) Modal Analysis: Review and Comparison, Archives of Computational Methods in Engineering 19 (1) (2012) 51-124.

[17] B. Peeters, G. d. Roeck, Stochastic System Identification for Operational Modal Analysis: A Review, Journal of Dynamic Systems, Measurement, and Control 123 (4) (2001) 659.

[18] R. Brincker, L. Zhang, P. Andersen, Modal identification of output-only systems using frequency domain decomposition, Smart Materials and Structures 10 (3) (2001) 441-445.

[19] R. Brincker, C. Ventura, P. Andersen, Damping Estimation by Frequency Domain Decomposition, in: Proceedings of the 19th International Modal Analysis Conference, 698-703, 2001.

20] N.-J. Jacobsen, P. Andersen, R. Brincker, Applications of Frequency Domain Curve-fitting in the EFDD Technique, in: Proceedings of the 26th International Modal Analysis Conference, 518-530, 2008.

[21] D. Tcherniak, S. Chauhan, M. H. Hansen, Applicability Limits of Operational Modal Analysis to Operational Wind Turbines, in: Proceedings of 28th International Modal Analysis Conference, 2010 .

[22] A. Benveniste, L. Mevel, Nonstationary Consistency of Subspace Methods, IEEE Transactions on Automatic Control 52 (6) (2007) 974-984.

[23] J. R. Wright, J. E. Cooper, Introduction to Aircraft Aeroelasticity and Loads, John Wiley, Chichester and England and and Hoboken, NJ, ISBN 978-0-470-85840-0, 2007.

[24] D. Tang, E. H. Dowell, Experimental and Theoretical Study on Aeroelastic Response of HighAspect-Ratio Wings, AIAA Journal 39 (8) (2001) 1430-1441.

[25] J. Heeg, Overview of the Aeroelastic Prediction Workshop, in: Proceedings of the 51st AIAA Aerospace Sciences Meeting, 1-21, 2013.

[26] C. Wieseman, P. Chwalowski, J. Heeg, A. Boucke, J. Castro, Structural Dynamics Modeling of HIRENASD in Support of the Aeroelastic Prediction Workshop, in: Proceedings of the 54th AIAA Structures, Structural Dynamics, and Materials Conference, 1-22, 2013.

[27] E. Neu, F. Janser, A. A. Khatibi, A. C. Orifici, Operational Modal Analysis of a Cantilever in a Wind Tunnel using Optical Fiber Bragg Grating Sensors, in: Proceedings of the 6th International Operational Modal Analysis Conference, 2015.

410 [28] C. R. Farrar, K. Worden, Structural Health Monitoring, John Wiley \& Sons, Ltd, Chichester, UK, 2012 .

[29] J. Lau, J. Debille, B. Peeters, S. Giclais, P. Lubrina, M. Boeswald, Y. Govers, Advanced systems and services for Ground Vibration Testing - Application for a research test on an Airbus A340-600 aircraft, in: Proceedings of the 15th International Forum on Aeroelasticity and Structural Dynamics, $1-10,2011$ 